\title{
INTERPROFISSIONALIDADE EM CUIDADOS PALIATIVOS: REFLEXÓES SOBRE A PRÁTICA
}

\author{
INTERPROFESSIONALITY IN PALLIATIVE CARE: REFLECTIONS ON \\ THE PRACTICE IN HEALTH CARE
}

Kelly Cristina Meller Sangoi, Lilian Zielke Hesler, Nadine Both da Silva, Tainá

Monique Schneider, Jenifer Rei da Silva, Daniela Pereira Gonzales \& Cristina

Scherbaum

${ }^{1}$ Universidade Regional Integrada do Alto Uruguai e das Missóes (URI), Santo Ângelo, RS, Brasil; ${ }^{2}$ Secretaria Municipal de Saúde do Município de Santo Ângelo, RS, Brasil.

\section{RESUMO}

Objetivo: descrever as ações do grupo de Cuidados Paliativos no domicílio do PET - Saúde/ Interprofissionalidade acerca da realização de um ciclo de palestras sobre os Cuidados Paliativos. Metodologia: Trata-se de um relato de experiência vivenciado por meio de um ciclo de palestras educativas sobre cuidados paliativos, direcionada a discentes, docentes, profissionais de saúde e comunidade, promovido pelo Programa de Educação pelo Trabalho para a Saúde (PET - Saúde/Interprofissionalidade) eixo Cuidados Paliativos no Domicílio. As palestras foram realizadas no segundo semestre do ano de 2019, na Universidade Regional Integrada do Alto Uruguai e das Missóes (URI), campus Santo Ângelo. Resultados: Os encontros foram organizados em 3 temáticas: comunicação de más notícias, cuidado paliativo em oncologia e critérios e indicações de cuidados paliativos. Através desta modalidade de ciclo de palestras foi possível proporcionar oportunidades para que os participantes pudessem adquirir conhecimentos com embasamento científico sobre a temática. Iniciar os cuidados paliativos o mais precoce possível é essencial para um melhor desfecho do quadro clínico, apesar do paciente não ter perspectiva de cura, iniciar os cuidados paliativos precocemente, auxilia no controle dos sintomas biopsíquicos contribuindo para uma melhor qualidade de vida dos mesmos e dos seus familiares. É essencial que atividades como educação em saúde, educação continuada, palestras e rodas de conversas sobre essa temática sejam disponibilizadas com frequência para que o tema se torne cada vez mais familiar e simples perante o olhar das pessoas, trazendo como consequência um cuidado mais qualificado diante do paciente que possui um prognóstico reservado.

Descritores: Cuidados paliativos; Educação continuada; Educação em saúde.

\section{ABSTRACT}

Objective: to describe the actions of the Palliative Care group at the home of PET - Health I Interprofessionality regarding the realization of a cycle of lectures on Palliative Care. Methodology: 
it is na account of experience lived through a cycle of educacional lectures on palliative care, animed at students, teachers, health professionals and the Community, promoted by the Education through Work for Health Program (PET - Saúde/Interprofissionalidade) Palliative Care at Home axis. The lectures were given in the second half of 2019, at the Integrated Regional University of Alto Uruguai $e$ das Missóes (URI), Santo Ângelo campus. Results: The meetings were organized into 3 themes: comunication of bad News, palliative care in oncology and palliative care criteria and indications. Through this type of lecture cycle, it was possible to provide opportunities for participants to acquire knowledge with a scientific basis on the subject. Starting palliative care as early as possible is essential for a better outcome of the clinical condition, although the patient has no prospect of a cure, starting palliative care early, helps in controlling biopsychic symptoms contributing to a better quality of life for them and their patients, Family members. It is essential that activities such as health education, continuing educations, lectures and rounds of conversations on this topic are made available frequently so that the topic becomes increasingly familiar and simple before the eyes of people, bringing as a consequence a more qualifies care in the face of the patient who has a poor prognosis.

Descriptors: Palliative care; Continuing education; Health education.

\section{INTRODUÇÁO}

O Programa de Educação pelo Trabalho para Saúde (PET) busca qualificar e proporcionar a integração entre ensino, serviços de saúde e a comunidade por meio de atividades educacionais envolvendo docentes e discentes dos cursos de graduação da área da saúde, profissionais da saúde dos municípios pertencentes ao programa e os usuários do Sistema Único de Saúde (SUS) ${ }^{1}$. Em sua nona edição, com início no ano de 2019, trouxe como tema central Educação Interprofissional em Saúde (EIP), em que participam 120 projetos do país com duração de 24 meses; os projetos contemplam ações de EIP na Atenção Básica $(\mathrm{AB})^{1}$.

A EIP é realizada quando discentes ou profissionais de profissóes distintas, aprendem com, a partir e sobre as profissóes, para assim prestar assistência qualificada aos usuários ${ }^{2}$. O trabalho interprofissional resulta em quebra de paradigma, prestando assistência aos usuários sem focar em áreas profissionais, estes tornando-se protagonistas dos serviços de saúde ${ }^{3}$.

O PET/saúde Interprofissionalidade realizado pela Universidade Regional Integrada Alto Uruguai e das Missóes (URI), campus Santo Ângelo em conjunto com a Secretaria Municipal de Saúde (SMS) de Santo Ângelo, contempla 5 eixos temáticos: eixo 1, Gestão em Saúde e Educação Permanente; eixo 2, Cuidados Paliativos no domicílio; eixo 3, Promoção da Saúde; eixo 4, Saúde do Trabalhador do SUS e eixo 5, Práticas Integrativas no SUS, com a participação dos seguintes cursos de graduação da Universidade: Enfermagem, Educação Física, Farmácia e Psicologia, com docentes e 
discentes, além dos preceptores que são profissionais da área da saúde que trabalham no SUS, em práticas gerenciais e assistenciais.

Nesta perspectiva, o Eixo 2 do PET Saúde/Interprofissionalidade - Cuidados Paliativos no domicílio tem como um de seus objetivos fortalecer o conceito de humanização do cuidado e o princípio da integralidade da assistência no contexto das redes colaborativas na formação para o SUS. Visa também promover espaços de responsabilidade, mudança de atitudes, desenvolver para além de competências técnicas, reconhecendo que a formação tem centralidade no desenvolvimento de valores para o trabalho em equipe ${ }^{1}$.

Cuidados Paliativos (CP) são os cuidados destinados a pessoas que possuem uma doença que ameace a continuidade da vida, visando melhorar a qualidade de vida dos mesmos e dos seus familiares e cuidadores, possibilitando alívio das dores e outros sintomas físicos e conforto no sofrimento psicossocial, auxiliando do início do tratamento até o luto ${ }^{4}$.

Através das diversas técnicas e percepções da equipe multiprofissional é possível prestar uma assistência mais qualificada, eficaz e abrangente, com abordagens farmacológicas e não farmacológicas, a fim de diminuir o sofrimento e proporcionar uma vida mais ativa aos pacientes e seus familiares ${ }^{6}$.

As atividades de educação em saúde têm sido utilizadas como uma importante ferramenta na promoção da saúde, a qual aproxima a prática profissional com a realidade de cada população, com ênfase na comunicação, informação e no desenvolvimento de habilidades, possibilitando assim, uma prática assistencial eficaz e integral ${ }^{7}$. Dentro dessa perspectiva, pensou-se em oferecer a discentes, docentes, profissionais da saúde e comunidade um ciclo de palestras que abordam a temática.

Sendo assim, o estudo tem por objetivo descrever as açóes do grupo de CP no domicílio do PET - Saúde/Interprofissionalidade acerca da realização de um ciclo de palestras sobre os Cuidados Paliativos.

\section{MÉTODO}

Trata-se de um estudo descritivo com abordagem qualitativa, do tipo relato de experiência. Descritiva, que tem a finalidade descrever particularidades de determinados indivíduos ou acontecimentos ${ }^{8}$ A abordagem qualitativa se aplica aos estudos de crenças, percepçóes, opinióes, relaçóes e interpretaçóes, com segmentos delimitados e focalizados ${ }^{9}$. Do tipo relato de experiência que busca a importância social da pesquisa, o desenvolvimento das relaçôes humanas e sociais, busca a vivência e a experiência de forma objetiva ${ }^{10}$. 
O estudo foi vivenciado pelo grupo, composto por sete discentes do curso de enfermagem, duas discentes do curso de psicologia, uma discente do curso de farmácia e um discente do curso de educaçáo física, em conjunto com duas docentes do curso de enfermagem e uma docente do curso de psicologia de uma instituiçáo de ensino superior privada, ainda em conjunto com três preceptoras, sendo elas, uma enfermeira, uma psicóloga e uma assistente social da SMS do município.

As vivências ocorreram no segundo semestre de 2019, em uma instituição de ensino superior, no interior do Estado do Rio Grande do Sul, através de três encontros com duração de duas horas.

\section{RESULTADOS E DISCUSSÓES}

Entre as estratégias de trabalho definidas pelo eixo 2 - CP no Domicílio para capacitação de tutores, preceptores, estudantes e profissionais de saúde foi realizar educação em saúde por meio de um Ciclo de Palestras aberto ao público. Foram realizados três encontros: "Comunicação de más notícias: é possível falar sobre isso?", "CP em Oncologia" e "Indicaçôes de CP", divulgadas nos serviços de saúde, universidade e redes sociais.

O primeiro encontro abordou o tema "Comunicação de más notícias: é possível falar sobre isso?”; ministrada por uma médica intensivista. Participaram do encontro aproximadamente 40 pessoas. Foi abordada a necessidade profissional em desenvolver habilidades de comunicação de más notícias, sendo esta qualquer notícia que modifique drástica e negativamente a visão do paciente sobre a continuidade da vida. As decisões compartilhadas entre paciente, família e equipe multiprofissional geram menos conflitos e culpa, além de propiciar o protagonismo do próprio paciente.

Para auxiliar a complexa atividade, foi exposto o protocolo Spikes, composto por seis etapas que planejam e organizam a transmissáo da má notícia, de forma fragmentada conforme a capacidade do interlocutor de assimilar as informaçóes transmitidas. $\mathrm{Na}$ transferência de informaçóes acerca do estado de saúde do paciente, deve-se ser honesto e verdadeiro, porém, ao mesmo tempo, não tirando a vontade do paciente viver e continuar sua vida, apesar de suas limitaçôes ${ }^{11,12}$.

Um estudo que objetivou descrever a compreensão dos acadêmicos e dos residentes de medicina ao lidar com a comunicação de más notícias e o impacto dessas notícias em suas vidas profissionais e pessoais, aponta que a comunicação de más notícias gera aflição e desconforto entre profissional e paciente, e ainda ressalta, a importância de preparar os profissionais de saúde desde a graduação, incluindo temas de comunicação com foco na transmissão de más notícias nas grades curriculares dos cursos da área da saúde, desta forma, beneficiaria tanto o profissional, como os pacientes e suas famílias ${ }^{13,14}$. 
A comunicação de más notícias é considerada como uma das práticas mais difíceis e delicadas das atividades dos profissionais da saúde. Por se tratar de um assunto complexo os profissionais devem adotar medidas para facilitar o entendimento e amenizar as angústias dos pacientes e dos seus familiares, como estabelecer uma comunicação calma, clara e objetiva e em um ambiente reservado além de demonstrar tranquilidade, sinceridade e empatia ${ }^{15,16}$.

Abordou-se no encontro seguinte a temática "CP em Oncologia" que foi ministrada por uma enfermeira residente em oncologia, onde aproximadamente 30 pessoas participaram.

$\mathrm{Na}$ maior parte dos indivíduos que apresentam o diagnóstico de câncer acabam recebendo essa informação em estágio avançado e incurável, fazendo com que o processo oncológico torne-se uns dos momentos em que promova abalos em diferentes aspectos, como psicológicos, sociais, econômicos, físicos, e principalmente emocionais, em que acabam refletindo e interferindo não apenas na vida do paciente mas também de sua família e cuidadores, na qual traz juntamente consigo uma bagagem de alteraçóes psíquicas, frustrações, medos e ansiedade ${ }^{17}$.

De tal forma, é de fundamental importância a atuação interprofissional para uma assistência integral ao paciente, pois ao realizar o cuidado na área de oncologia não trata-se apenas de um âmbito limitado, mas sim da realização da promoção da assistência holística, visando o bem-estar geral do paciente, uma vez que a situaçáo em que o mesmo encontra-se é em um estado de fragilidade e vulnerabilidade, consequentemente requer que não haja apenas a ação da assistência a cura da doença, mas que também haja um vínculo para proporcionar ao paciente o conforto, a qual o desempenho interprofissional é indispensável para a prática do cuidado à saúde ${ }^{18}$.

Discutiu-se também na proposta de um organograma as referências e contrareferências para atenção ao paciente oncológico, modalidades de tratamento, sendo estes, curativos ou paliativos. Enfatizou a comunicação eficaz da equipe, paciente e família, a fim de apoiá-lo, garantindo seus direitos, sempre respeitando suas condiçóes biopsicossociais. Destacou-se ainda a importância de prevenir sintomas que provavelmente irão prejudicar, inquietar e de certa forma preocupar o paciente acerca do seu tratamento, onde ele pode vir a se fazer questionamentos equivocados. Deve-se identificar precocemente alguns sintomas, como por exemplo dor, náuseas e enjoo, e então prescrever cuidados que auxiliem na melhora clínica, e consequentemente emocional.

Segundo Silva e Sudigurski ${ }^{18}$, é dever dos profissionais da saúde proporcionar alívio e controle da dor e demais sintomas físicos, psíquicos, sociais e espirituais aos pacientes em cuidados paliativos, a fim de amenizar o sofrimento e proporcionar um cuidado integral aos mesmos. 
$\mathrm{Na}$ realidade, os pacientes que estão em estado avançado, percebem que sua vida está chegando ao fim, mesmo que ninguém os tenha dito nada, e assim, eles se expressam através de sinais, mensagens ou alguma forma diferente, e os profissionais da área da saúde, devem prestar atenção a estes, para que suas vontades e desejos sejam atendidos na medida do possível, para diminuir sua inquietação e angústia, e assim, trazer maior tranquilidade e paz para o processo de morrer ${ }^{19}$.

Iniciar precocemente os cuidados paliativos na oncologia auxilia numa melhor qualidade de vida aos pacientes, pois desta forma é possível prevenir e controlar os mais diversos sintomas que estão associados a patologia, bem como ao tratamento que possivelmente o paciente está sendo submetido.

No último encontro do ciclo de palestras versou-se sobre "Critérios e Indicações de CP”, que foi ministrada por uma médica geriatra e paliativistas, integrante do núcleo de Cuidados Paliativos em um hospital privado. Participaram desse encontro aproximadamente 30 pessoas.

Discutiu-se sobre os conceitos de CP, uma abordagem de tratamento multidisciplinar utilizada para doenças que ameaçam a continuidade da vida, abrangendo também sua família. Entre seus principais objetivos estão a melhorar a qualidade de vida e influenciar positivamente o curso da doença promovendo o alívio da dor e os demais sintomas excruciantes buscando assim proporcionar uma maneira de auxílio, para garantir que o indivíduo que possui a patologia possa viver ativamente até a sua finitude, em que a mesma deve ser encarada como um processo natural da vida ${ }^{18}$.

De acordo com o Manual de Cuidados Paliativos ${ }^{6}$, às práticas paliativas consistem em diferentes pontos de atuação, tendo as mais variadas especialidades adquirindo assim o conhecimento em que favorece a assistência do cuidado ao paciente e do processo da doença, promovendo possibilidades de intervençóes clínicas e terapêuticas da área da saúde, proporcionando um cuidado individual e de conhecimento específico, de acordo com que o paciente necessita naquele exato momento. Para isso, consequentemente buscam-se contínuas estratégias de apoios, como escalas que venham a ajudar na indicação de cuidados paliativos, sinais e sintomas que venham possibilitar a identificação de manifestações do processo da doença em fases iniciais, possibilitando assim controle precoce da patologia aos indivíduos que apresentam suas chances de intervençóes de cura do tratamento diminuídas ou prolongamento da vida em que se percebe e demonstram padecimento na forma psíquica, espiritual ou físico proporciona-se a escolha da prática do cuidado, do conforto e dignidade de vida objetivando seu bem-estar.

A Academia Nacional de Cuidados Paliativos ${ }^{6}$, prevê algumas morbidades como critérios de indicação para cuidados paliativos, como: Esclerose Lateral Amiotrófica (ELA), HIV- Síndrome da Imunodeficiência Adquirida (SIDA), Doença Pulmonar Obstrutiva Crônica (DPOC), demências, doenças degenerativas progressivas, hepatites, 
cânceres, entre outras. Cabe ressaltar que estas morbidades não têm possibilidade de cura e ameaçam a continuidade da vida ${ }^{20}$.

Foram apresentadas escalas que podem auxiliar na indicação de cuidados paliativos e na tomada de decisões, bem como podem auxiliar na avaliação clínica e funcional dos pacientes; entre estas, a Escala de Atividades de Vida Diária, Escala de Karnofsky, Escala de Performance Paliativa, e a Escala de Avaliação de Sintomas de Edmonton, a utilização dessas escalas são medidas relacionadas à tentativa de quantificar o bem-estar geral dos pacientes. As escalas são ferramentas utilizadas para elegibilidade deste fim, por ser um instrumento validado que permite a avaliação da funcionalidade e a compreensão da trajetória da doença de base ${ }^{4,6}$.

Iniciar os cuidados paliativos o mais precoce possível é essencial para um melhor desfecho do quadro clínico, apesar dos pacientes não ter perspectiva de cura, iniciar os cuidados paliativos precocemente auxilia no controle dos sintomas biopsíquicos, contribuindo para uma melhor qualidade de vida dos mesmos e dos seus familiares.

\section{CONSIDERAÇÓES FINAIS}

O CP é um tema pouco abordado, visto como tabu na cultura ocidental. Nessa perspectiva, através da educação em saúde proporcionada pelo ciclo de palestras, foi possível proporcionar oportunidades para que os participantes pudessem adquirir conhecimentos com embasamento científico.

Perante esse contexto podemos observar com a participação dos alunos, tutores e profissionais da saúde de diversas áreas, o quão importante é a relação e conexão interprofissional, que auxiliam na agregação de conhecimentos variados, observando e trabalhando com o tema de maneira mais ampla e consequentemente, ajudando o paciente, familiar e cuidador de forma integral.

Portanto, é essencial que atividades como educação em saúde, educação continuada, palestras e rodas de conversas sobre CP sejam proporcionados com frequência para que a temática seja difundida na academia e aos profissionais da saúde, trazendo como consequência um cuidado qualificado diante do paciente que possui um prognóstico reservado.

\section{REFERÊNCIAS}

Brasil. Ministério da Saúde (MS). PET-Saúde/Interprofissionalidade inicia atividades da nona edição. Brasília DF. Abr., 2019. Disponível em: https:/www.saude.gov.br/ noticias/sgtes/45332-pet-saude-interprofissionalidade-inicia-atividades-da-nona-edicao 
Barr, H; Low, H. Introdução à Educação Interprofissional. Centro para o Avanço da Educação Interprofissional. 2013.

Brasil. Ministério da Saúde (MS). Cuidados Paliativos. Brasília DF. 2017. Disponível em: https://telessaude.hc.ufmg.br/wp-content/uploads/2016/07/CUIDADOSPALIATIVOS_LIVRO.pdf.

Brasil. Ministério da Saúde/ Secretaria de Gestão do Trabalho e da Educação em Saúde. Departamento de Gestão da Educação na Saúde. Relatório Final da Oficina de Alinhamento Conceitual sobre Educação e Trabalho Interprofissional em Saúde. Brasília DF. 2017.

Brasil. Ministério da Saúde (MS). Ministério da Saúde normatiza cuidados paliativos no SUS. Brasília DF. Nov., 2018. Disponível em: https://www.saude.gov.br/noticias/ agencia-saude/44723-ministerio-normatiza-cuidados-paliativos-no-sus .

Academia Nacional de Cuidados Paliativos (ANCP). Manual de Cuidados Paliativos. ed. 2, ago., 2019. Disponível em: http://biblioteca.cofen.gov.br/wp-content/ uploads/2017/05/Manual-de-cuidados-paliativos-ANCP.pdf .

Salci, M. A. et al. Educação em saúde e suas perspectivas teóricas: algumas reflexóes. Texto Contexto Enferm. Florianópolis. v. 22, n. 1, p. 224-30, Jan./Mar., 2013. Disponível em:<http://www.scielo.br/scielo.php?script=sci_arttext\&pid=S010407072013000100027\&lng=en\&nrm=iso\&tlng=pt.

Gil, A. C. Como elaborar projetos de pesquisa. São Paulo. Atlas. 4o ed. p.17-57. 2002. Disponível em: http://home.ufam.edu.br/salomao/Tecnicas\%20de\%20Pesquisa\%20 em\%20Economia/Textos\%20de\%20apoio/GIL,\%20Antonio\%20Carlos\%20\%20 Como\%20elaborar\%20projetos\%20de\%20pesquisa.pdf.

Minayo, M.C.S. O desafio do conhecimento: pesquisa qualitativa em saúde. São Paulo SP. Hucitec Editora, 140 ed., 2014.

Minayo, M.C.S. Pesquisa social: teoria, método e criatividade. Petrópolis: Vozes. 28 a ed. 2009.

Cruz, C. O.; Riera, R. Comunicando más notícias: o protocolo SPIKES. Diagn Tratamento. v. 21, n. 3, p. 106-108, 2016. Disponível em: http://docs.bvsalud.org/ biblioref/2016/08/1365/rdt_v21n3_106-108.pdf.

Koch, C. L.; Rosa, A. B.; Bedin, S. C. Más notícias: significados atribuídos na prática assistencial neonatal/pediátrica. Revista Bioética. v. 25, n. 3, p. 577-584, 2017. Disponível em: http://www.scielo.br/scielo.php?script=sci_arttext\&pid=S198380422017000300577\&lang=pt 
Vogel, K. P. et al. Comunicação de Más Notícias: Ferramenta Essencial na Graduação Médica. Rev. Bras. Educ. Med.. v. 43, n.1, p. 314-321, 2019.

Disponível em: http://www.scielo.br/scielo.php?script=sci_arttext\&pid=S0100$55022019000500314 \&$ lang=pt

Andrade, C. G. D. CUIDADOS PALIATIVOS: comunicação entre enfermeiro e paciente terminal. Repositório Institucional da UFPB, João Pessoa, mar./2015. Disponível em: https://repositorio.ufpb.br/jspui/handle/tede/5124.

Neto, J. A. C.; et al. Profissionais de saúde e a comunicação de más notícias sob a ótica do paciente. Rev Med Minas Gerais. v. 23, n. 4, p. 518-525, 2013. Disponível em: http://rmmg.org/exportar-pdf/415/v23n4a14.pdf

Lima, K. M. A.; Maia, A. H. N.; Nascimento, I. R. C. Comunicação de más notícias em cuidados paliativos na oncopediatria. Revista Bioética. v. 27, n. 4, p. 719-727, 2019. Disponível em: http://www.scielo.br/scielo.php?script=sci_arttext\&pid=S198380422019000400719\&lang=pt

SILVA, R.C.F.; HORTALE, V.A. Cuidados paliativos oncológicos: elementos para o debate de diretrizes nesta área. Cad. Saúde Pública. 2016, vol.22, n.10, pp.2055-2066. ISSN 0102-311X. Disponível em: https://doi.org/10.1590/S0102$311 \mathrm{X} 2006001000011$.

Silva, E. P.; Sudigurski, D. Concepções sobre cuidados paliativos: revisão bibliográfica. Acta Paul Enferm. v. 21, n. 3, p.504-508, 2018. Disponível em: https://www. researchgate.net/publication/250049611_Concepcoes_sobre_cuidados_paliat0ivos_ revisao_bibliografica .

Siqueira, C. L., Campos J. C. Comunicação de más notícias por enfermeiras de oncologia na ótica da Teoria Humanística de Enfermagem. CIAIQ2018, v. 2, jul./2018. Disponível em: https://www.proceedings.ciaiq.org/index.php/ciaiq2018/ article/view/1821.

Bravalhieri, A. A. V. et al. Características de pacientes com indicação de cuidados paliativos em uma unidade de cuidados prolongados em Campo Grande, Mato Grosso do Sul. Revista Multitemas, v. 25, n. 59, p. 211-226, mar. /2020. Disponível em: https://multitemas.ucdb.br/multitemas/article/view/2878.

Autor Correspondente: Kelly Cristina Meller Sangoi E-mail: kellysangoi@gmail.com

Recebido em: 2020-08-25

Aprovado: 2020-12-10 